\title{
ESTIMATIVA DE PRODUTIVIDADE DA CULTURA DO FEIJÃO IRRIGADO EM CRISTALINA-GO, PARA DIFERENTES LÂMINAS DE IRRIGAÇÃO COMO FUNÇÃO DA UNIFORMIDADE DE APLICAÇÃO
}

\author{
EVERARDO C. MANTOVANI ${ }^{1}$, DAVID R. P. MONTES ${ }^{2}$, GUSTAVO H. S. VIEIRA ${ }^{3}$, \\ MÁRCIO M. RAMOS ${ }^{4}$, ANTÔNIO A. SOARES ${ }^{5}$
}

\begin{abstract}
RESUMO: O estudo foi realizado por meio de simulações, inseridas em seis cenários de níveis de uniformidade de aplicação de água, nas condições de Cristalina-GO, com o objetivo de avaliar o efeito da aplicação de diferentes lâminas de irrigação na produtividade comercial da cultura do feijão (Phaseolus vulgaris L.) cv. Carioca. Para tanto, utilizou-se o sistema de irrigação por aspersão convencional, mediante dados de produtividade obtidos por intermédio da função de produtividade desenvolvida para as condições locais. Os resultados obtidos permitiram concluir que a maior produtividade de grãos $\left(2.946,52 \mathrm{~kg} \mathrm{ha}^{-1}\right.$ ) foi obtida com o maior coeficiente de uniformidade de aplicação da água do sistema de irrigação por aspersão (90\%), que resultou no menor valor de lâmina média coletada $(418,16 \mathrm{~mm})$ no ciclo da cultura. Ainda foi possível observar que o modelo e o método adotados se mostraram adequados para gerar cenários de produtividade do feijoeiro para as condições locais do presente estudo.
\end{abstract}

PALAVRAS-CHAVE: Phaseolus vulgaris, irrigação, função de produtividade, aspersão, eficiência de irrigação.

\section{ESTIMATED PRODUCTION OF BEAN CROP IRRIGATED IN CRISTALINA-GO UNDER DIFFERENT DEPTHS AND UNIFORMITY OF APPLICATION}

\begin{abstract}
The study was carried out with simulations for six scenarios of water application uniformity levels under the conditions of Cristalina-GO (Brazil), in order to evaluate the effect of different irrigation levels in the commercial yield of beans (Phaseolus vulgaris L.) cv. Carioca. For this it was used the conventional sprinkler irrigation system, using productivity data obtained with the production function developed for local conditions. The results showed that the highest grain yield $\left(2,946.52 \mathrm{~kg} \mathrm{ha}^{-1}\right)$ was obtained with the highest coefficient of water application uniformity in sprinkler irrigation system $(90 \%)$ which resulted in the lowest average depth collected (418.16 mm) in the crop cycle. It was even observed that the model and the method adopted proved to be adequate to generate scenarios of bean yield to local conditions of the present study.
\end{abstract}

KEYWORDS: Phaseolus vulgaris, irrigation, production function, sprinkler irrigation, irrigation efficiency.

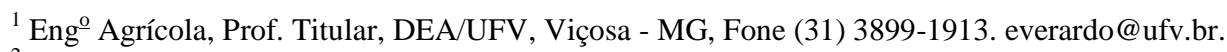

${ }^{2}$ Eng ${ }^{0}$ Agrícola, Doutorando em Engenharia Agrícola, DEA/UFV, d_palomino13@ @otmail.com.

${ }^{3}$ Eng ${ }^{\text {o }}$ Agrônomo, Doutorando em Engenharia Agrícola, DEA/UFV, Prof. IFES, campus Santa Teresa, ghsvieira@ gmail.com.

${ }^{4}$ Eng ${ }^{\circ}$ Agrônomo, Prof. Titular DEA/UFV, Viçosa - MG, mmramos@ ufv.br.

${ }^{5}$ Eng $^{\mathrm{o}}$ Agrícola, Prof. Titular, DEA/UFV, Viçosa - MG, aasoares@ufv.br.

Recebido pelo Conselho Editorial em: 9-7-2010

Aprovado pelo Conselho Editorial em: 27-9-2011
} 


\section{INTRODUÇÃO}

O uso racional da água na irrigação implica reduzir as perdas por evaporação, escoamento superficial, percolação, dentre outras (OLIVEIRA \& FIGUEIREDO, 2007), e um dos aspectos mais importantes a serem observados, no manejo da irrigação, é a uniformidade de distribuição de água pelo sistema (JENSEN, 2007). ROCHA et al. (1999) destacaram que o coeficiente de uniformidade é influenciado, principalmente, pelo espaçamento entre aspersores, velocidade do vento e pressão de serviço.

Segundo FARIA et al. (2009), a distribuição da água aplicada dos sistemas de aspersão é um processo que passa por duas etapas: aplicação da água na superfície do solo ou da cultura e redistribuição da água aplicada no interior do solo. Apesar dos efeitos positivos da redistribuição da água no solo, que, em alguns casos, pode anular os efeitos da desuniformidade de aplicação, deve-se considerar, conforme enfatizado por FRIZZONE et al. (2007), que a uniformidade do teor de água do solo e a produtividade das culturas irrigadas são bastante dependentes da uniformidade com que a água é aplicada durante a primeira fase desse processo.

Segundo HSIAO et al. (2007), a melhoria da performance dos sistemas de irrigação e o aprimoramento do manejo da irrigação são de grande importância e são necessários para aumentar a produtividade por unidade de água e tornar a agricultura irrigada sustentável, uma vez que a disponibilidade de água para a irrigação será reduzida no futuro, devido ao aumento da demanda por outros setores prioritários (SANTOS et al., 2010).

A água é um dos principais fatores de produção na agricultura, e cada espécie de planta necessita de um adequado nível de água no solo para que suas necessidades fisiológicas sejam atendidas (FREITAS et al., 2003). SANTANA et al. (2008) afirmaram que a irrigação constitui a alternativa viável, dentre os tratos culturais, na melhoria da produtividade, e que sua finalidade básica é proporcionar água à cultura para atender à sua exigência hídrica.

As funções de produtividade são modelos que permitem estimar a produtividade de uma cultura em diferentes cenários, e vários deles relacionam a uniformidade de irrigação e a produtividade de determinada cultura (REZENDE et al., 2004). De acordo com BERNARDO (2006), define-se como função de produtividade a relação técnica entre um conjunto específico de fatores envolvidos num processo produtivo qualquer e a produtividade física possível de se obter com a tecnologia existente. MANTOVANI et al. (1995) definiram função de produtividade como a relação que caracteriza a resposta da cultura a determinados fatores, como água, fertilizante e energia, em que o conhecimento destas relações é necessário para avaliação de estratégias de irrigação.

De acordo com MANTOVANI et al. (2009b), há três tipos de modelagens das funções de produtividade água-cultura: transpiração-cultura, evapotranspiração-cultura e água-cultura. As primeiras pesquisas sobre a relação água-planta apresentaram estimativas da produção de matéria seca em função da transpiração. Contudo, devido à maior facilidade de se medir a evapotranspiração em campo, foram desenvolvidas muitas aproximações com base nesta variável.

DOORENBOS \& KASSAM (1979) adotaram o modelo desenvolvido por Stewart (eq.(1)) para quantificar a relação entre a produtividade das culturas e a quantidade de água aplicada, com base na relação evapotranspiração-cultura.

$$
1-\frac{\mathrm{P}}{\mathrm{P}_{\mathrm{MAX}}}=\beta\left(1-\frac{\mathrm{ET}}{\mathrm{ET}_{\mathrm{MAX}}}\right)
$$

em que,

$\mathrm{P}$ - produtividade, $\mathrm{kg} \mathrm{ha}^{-1}$;

$\mathrm{P}_{\mathrm{MAX}}$ - produtividade máxima, $\mathrm{kg} \mathrm{ha}^{-1}$;

$\beta$ - coeficiente de sensibilidade da cultura ao déficit hídrico;

ET - evapotranspiração sazonal da cultura, mm, e

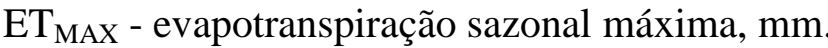


Segundo MANTOVANI et al. (1995), a relação entre rendimento e irrigação não é linear, ao contrário da relação entre rendimento e evapotranspiração, uma vez que a água aplicada não é totalmente utilizada pela cultura. Assim, a função depende da uniformidade de distribuição de água. Neste sentido, o autor incorporou ao modelo de Stewart uma função que relaciona a evapotranspiração com a quantidade de água aplicada na irrigação e sua uniformidade de aplicação.

De acordo com BINOTTI et al. (2007), a partir da década de 1980, o feijoeiro passou a ser cultivado também na época de inverno sob irrigação, atraindo médios e grandes produtores. Neste sentido, o agricultor é estimulado a utilizar maiores níveis tecnológicos, obtendo produtividades de três a cinco vezes superiores às obtidas em sequeiro, devido a ser uma cultura altamente responsiva à irrigação (EFETHA et al., 2011) e altamente eficiente no uso da água, chegando a produzir em torno de $10 \mathrm{~kg} \mathrm{ha}^{-1} \mathrm{~mm}^{-1}$ (MUNOZ-PEREA et al., 2007).

Neste contexto, o objetivo do presente trabalho foi simular o efeito da aplicação de diferentes lâminas de irrigação obtidas em função de seis níveis de uniformidade de aplicação de água de um sistema de irrigação por aspersão convencional na produtividade da cultura do feijão (Phaseolus vulgaris L.) mediante utilização de função de produtividade.

\section{MATERIAL E MÉTODOS}

O estudo foi realizado por meio de simulações efetuadas para as condições de Cristalina-GO, que se localiza na latitude $16^{\circ} 45^{\prime} \mathrm{S}$ e na longitude $47^{\circ} 36^{\prime} \mathrm{W}$, com altitude de $850 \mathrm{~m}$. O solo predominante na região é da classe Latossolo Vermelho-Amarelo. Segundo a classificação climática proposta por Köeppen, Cristalina possui um clima do tipo Cwa, isto é, tropical de altitude, com chuva nos meses de outubro a maio, e com período seco de junho a setembro. A região apresenta precipitação pluviométrica anual de $1.600 \mathrm{~mm}$, temperatura média anual de $20,5^{\circ} \mathrm{C}$ e umidade relativa em torno de $40 \%$ no inverno e $70 \%$ no verão (SCHMIDT \& VALIATI, 2006). Nessas condições, foi estimada a produtividade da cultura do feijão (Phaseolus vulgaris L.) $c v$. Carioca, utilizando-se de um polinômio de segundo grau (eq.(2)) como modelo de ajuste da função de produtividade.

$$
y=f(w)=a w^{2}+b w+c
$$

em que,

$\mathrm{y}$ - produtividade, $\mathrm{kg} \mathrm{ha}^{-1}$;

w - lâmina total de água aplicada, mm, e

a, b e c - parâmetros do modelo.

Para a estimativa da produtividade, utilizou-se o modelo proposto por FIGUERÊDO et al. (2008), que trabalharam no campo experimental da Embrapa Cerrados, em clima e solos semelhantes ao estudado neste trabalho, determinando a função de produtividade para a cultura do feijão $c v$. carioca, no período seco. Esta função considera as lâminas totais de água aplicadas por um sistema de irrigação com $88 \%$ de eficiência de irrigação. A função de produtividade utilizada no presente estudo (eq.(3)) considera somente as lâminas de irrigação aplicadas.

$$
y=-0,0181 w^{2}+16,628 w-791,37
$$

em que,

$\mathrm{y}$ - produtividade, $\mathrm{kg} \mathrm{ha}^{-1}$, e

w - lâmina total de água aplicada, mm.

A estimativa da produtividade do feijão $c v$. Carioca, nas condições de Cristalina-GO, foi realizada para o período seco do ano, isto é, outono/inverno, utilizando-se de um espaçamento 0,50 m entre fileiras de plantio, e uma densidade de 12 plantas por metro linear (MENDONÇA et al., 2007; SANTANA et al., 2008), sob irrigação por aspersão convencional, com aspersores e linhas laterais espaçados em 18 x $18 \mathrm{~m}$. As características físico-hídricas utilizadas na simulação do 
programa foram as de um solo com $0,40 \mathrm{~m}$ de profundidade efetiva. Consideraram-se $33,3 \%$ e $22,3 \%$, com base em massa, como valores médios de capacidade de campo e de ponto de murchamento, respectivamente. A densidade do solo adotada foi de $1,2 \mathrm{~g} \mathrm{~cm}^{-3}$, com as respectivas porcentagens de argila, silte e areia iguais a 50; 30 e $20 \%$.

O tempo de avaliação correspondeu ao período vegetativo do feijoeiro, iniciando-se no dia de plantio da cultura (primeiro de julho de 2009). A duração de cada fase fenológica foi definida em função dos graus-dia (eq.(4)), conforme metodologia de Arnold, apresentada por LIMA \& SILVA (2008), de modo que a planta atingisse determinado somatório térmico a partir da temperatura basal favorável ao seu desenvolvimento. O valor adotado como temperatura basal do feijoeiro foi de $13{ }^{\circ} \mathrm{C}$.

$$
\mathrm{GD}=\frac{\left(\mathrm{T}_{\text {máx }}-\mathrm{T}_{\text {mín }}\right)}{2}-\mathrm{T}_{\text {basal }}
$$

em que,

GD - graus-dia;

$\mathrm{T}_{\text {máx }}$ - temperatura máxima diária do ar, ${ }^{\circ} \mathrm{C}$;

$\mathrm{T}_{\text {mín }}$ - temperatura mínima diária do ar, ${ }^{\circ} \mathrm{C}$, e

$\mathrm{T}_{\text {basal }}$ - temperatura abaixo da qual as plantas não se desenvolvem, ${ }^{\circ} \mathrm{C}$.

A duração das fases vegetativas do feijoeiro, em função dos graus-dias; assim como os valores diários de coeficiente de cultura $(\mathrm{Kc})$ obtidos por interpolação entre os valores de referência, foram determinados por meio da simulação com o software IRRIPLUS. Em função dos graus-dia, o período total de avaliação correspondente ao ciclo da cultura, para as condições edafoclimáticas da região, foi de 92 dias (820,4 graus-dia), desde o plantio até a colheita, com 28 dias (193,7 graus-dia) de duração na fase inicial, 26 dias (227,5 graus-dia) na de crescimento, 28 dias (296,5 graus-dia) na de produção e 10 dias (102,7 graus-dia) na de maturação, com valores médios de Kc para cada um desses períodos iguais a 0,$5 ; 0,7 ; 1,25$ e 0,6, respectivamente. MANTOVANI (1986) encontrou, para o feijoeiro, valores de Kc iguais a 0,61; 0,80; 1,37 e 0,65, para o estágio inicial, intermediário, de crescimento e final, respectivamente, nas condições de Viçosa-MG. SANTANA et al. (2008) apresentaram valores de Kc iguais a 0,53; 0,81; 1,07 e 0,78 para o estádio inicial, intermediário, de crescimento e final, respectivamente para o feijoeiro, nas condições de Patos de Minas-MG. Já DOORENBOS \& KASSAM (1979) apresentaram valores de 0,$40 ; 0,80 ; 1,20$ e 0,75 para condições de umidade relativa menor que $20 \%$ e vento forte.

A evapotranspiração de referência (eq.(5)) diária foi obtida pelo método de Penman-Monteith (PEREIRA et al., 2010), utilizando-se do histórico de 17 anos de dados climáticos médios diários da região de estudo, registrados pelo Instituto Nacional de Meteorologia (INMET) e cadastrados na base do software IRRIPLUS. O programa possui rotina de cálculo, que estima o saldo de radiação $(\mathrm{Rn})$ a partir de valores de duração do brilho solar $(\mathrm{h})$, temperatura $\left({ }^{\circ} \mathrm{C}\right)$ e umidade relativa (\%), e dados locais de latitude, longitude e altitude (m). A densidade do fluxo de calor do solo (G) foi considerada igual a zero para o cálculo na base diária.

$$
\text { ETo }=\frac{0,408 \Delta\left(\mathrm{R}_{\mathrm{n}}-\mathrm{G}\right)+\gamma \frac{900}{\mathrm{~T}+273} \mathrm{u}_{2}\left(\mathrm{e}_{\mathrm{s}}-\mathrm{e}_{\mathrm{a}}\right)}{\Delta+\gamma\left(1+0,34 \mathrm{u}_{2}\right)}
$$

em que,

ETo - evapotranspiração de referência, $\mathrm{mm} \mathrm{dia}^{-1}$;

$\mathrm{R}_{\mathrm{n}}$ - saldo de radiação na superfície da cultura, $\mathrm{MJ} \mathrm{m}^{-2} \mathrm{dia}^{-1}$;

$\mathrm{G}$ - densidade do fluxo de calor do solo, $\mathrm{MJ} \mathrm{m}^{-2} \mathrm{dia}^{-1}$;

$\mathrm{T}$ - temperatura do ar média diária a $2 \mathrm{~m}$ de altura, ${ }^{\circ} \mathrm{C}$;

$\mathrm{u}_{2}$ - velocidade do vento a $2 \mathrm{~m}$ de altura, $\mathrm{m} \mathrm{s}^{-1}$; 
$\mathrm{e}_{\mathrm{s}}$ - pressão de vapor de saturação, $\mathrm{kPa}$;

$\mathrm{e}_{\mathrm{a}}$ - pressão parcial de vapor, $\mathrm{kPa}$;

$\mathrm{e}_{\mathrm{s}}-\mathrm{e}_{\mathrm{a}}$ - déficit de pressão de vapor de saturação, $\mathrm{kPa}$;

$\Delta$ - declividade da curva de pressão de vapor, $\mathrm{kPa}^{\circ} \mathrm{C}^{-1}, \mathrm{e}$

$\gamma$ - coeficiente psicrométrico, $\mathrm{kPa}^{\circ} \mathrm{C}^{-1}$.

Os valores diários de evapotranspiração da cultura (ETc) foram obtidos pelo produto entre o coeficiente da cultura $(\mathrm{Kc})$, o coeficiente de umidade do solo $(\mathrm{Ks})$ pelo método logarítmico e a ETo, de acordo com o método FAO (eq.(6).

$\mathrm{ETc}=\mathrm{Kc} \mathrm{Ks} \mathrm{ETo}$

em que,

ETc - evapotranspiração da cultura, $\mathrm{mm} \mathrm{dia}^{-1}$;

Kc - coeficiente da cultura;

Ks - coeficiente de déficit de água no solo, e

ETo - evapotranspiração de referência, $\mathrm{mm} \mathrm{dia}^{-1}$.

As lâminas líquidas de irrigação foram determinadas com base nos valores encontrados de ETc durante o ciclo da cultura, com aplicação das lâminas em um turno de rega variável, de 4 a 8 dias, determinado em função da capacidade total de armazenamento de água no solo (CTA = $68,3 \mathrm{~mm}$ ) e o fator de disponibilidade hídrica para o feijoeiro $(\mathrm{f}=0,4)$. Simularam-se, no total, 18 eventos de irrigação, que foram interrompidos dois dias antes da colheita.

Para o cálculo da produtividade da cultura, não se consideraram as chuvas, devido ao período em que a cultura foi manejada, correspondente à época de inverno, caracterizada pela ausência de precipitações na maioria dos anos. É neste período que o feijão tem maior aceitação entre os médios e grandes produtores, pela obtenção de produtividades superiores em comparação com outras épocas do ano (BINOTTI et al., 2007).

Na determinação da produtividade do feijoeiro, foi realizada a simulação da irrigação dentro de uma área de $324 \mathrm{~m}^{2}$, relativa à superposição da precipitação dos aspersores espaçados de 18 x $18 \mathrm{~m}$. Dentro da área, simulou-se a lâmina coletada total em todo o ciclo da cultura (w), em cada um dos 36 pluviômetros coletores. Simulou-se a disposição dos coletores numa área unitária de $3 \mathrm{~m} \times 3 \mathrm{~m}$, representando, cada um, 1/36 da área total. Nesta análise, foi considerada uma área adequadamente irrigada de $80 \%$ e trabalhou-se com seis valores de uniformidade de aplicação de água do sistema de irrigação, definidos pelo coeficiente de uniformidade de Christiansen (CUC) (MANTOVANI et al., 2009a), sendo avaliados os valores de 65; 70; 75; 80; 85 e 90\%.

No cálculo da lâmina coletada total sazonal em cada pluviômetro coletor (w), foi gerado manualmente um cenário, simulando-se valores de lâminas coletadas em cada um dos 36 pluviômetros da área de $324 \mathrm{~m}^{2}$, para a obtenção dos valores de CUC preestabelecidos. Desta forma, realizou-se uma análise de distribuição normal com o valor de CUC preestabelecido e um valor assumido de lâmina média coletada pelos pluviômetros.

Por meio do valor da lâmina de irrigação necessária, em todo o ciclo da cultura (IRN), foi determinada, para cada valor de CUC, a lâmina de irrigação total necessária (ITN) que representa a lâmina média aplicada no ciclo da cultura (eq.(7)). Para tanto, considerou-se a eficiência de aplicação do sistema de irrigação, em $80 \%$ de área adequadamente irrigada (eq.(8)). Essa eficiência foi calculada por meio do valor da eficiência de distribuição $\left(\mathrm{ED}_{80}\right)$ e a eficiência de aplicação em potencial (EAp). Os valores de $\mathrm{ED}_{80}$, para cada nível de CUC, foram determinados a partir dos valores de lâmina gerados pela análise de distribuição normal, utilizando-se da relação entre a lâmina mínima aplicada em $80 \%$ da área e a lâmina média preestabelecida, conforme metodologia apresentada por KELLER \& BLIESNER (1990). 


$$
\begin{aligned}
& \mathrm{ITN}=\frac{\mathrm{IRN}}{\mathrm{EA}_{80}} \\
& \mathrm{EA}_{80}=\mathrm{ED}_{80} \mathrm{EA}_{\mathrm{p}}
\end{aligned}
$$

em que,

ITN - irrigação total necessária, mm;

IRN - irrigação real necessária, mm;

$\mathrm{EA}_{80}$ - eficiência de aplicação em $80 \%$ de área adequadamente irrigada, \%;

$\mathrm{ED}_{80}$ - eficiência de distribuição em $80 \%$ de área adequadamente irrigada, \%, e

EAP - eficiência de aplicação em potencial.

O valor da eficiência de aplicação em potencial (EAp) foi calculado por intermédio da eq.(9), desenvolvida por Keller e apresentada por BERNARDO et al. (2008). Essa característica está em função do índice de pulverização do jato do aspersor (CI), definido pela eq.(10). Tal índice refere-se ao aspersor da marca Fabrimar, modelo ECO A232, com diâmetros de bocais de 6,6 mm x 4,0 mm, trabalhando com pressão de serviço de $250 \mathrm{kPa}$, vazão de $3,5 \mathrm{~m}^{3} \mathrm{~h}^{-1}$ e raio de alcance de $16 \mathrm{~m}$.

$$
\begin{aligned}
& \mathrm{EA}_{\mathrm{P}}=0,976+0,005 \text { ETo }-0,00017 \mathrm{ETo}^{2}+0,0012 \mathrm{Vv}- \\
& \mathrm{CI}(0,00043 \mathrm{ETo}+0,00018 \mathrm{Vv}+0,000016 \mathrm{ETo} \mathrm{Vv}) \\
& \mathrm{CI}=0,032 \mathrm{P}^{1,3} \mathrm{DB}^{-1}
\end{aligned}
$$

em que,

EA $A_{P}$ - eficiência de aplicação em potencial;

ETo - evapotranspiração de referência máxima, $\mathrm{mm} \mathrm{dia}^{-1}$;

Vv - velocidade do vento da série histórica, $\mathrm{km} \mathrm{dia}^{-1}$;

CI - índice de pulverização do jato do aspersor;

$\mathrm{P}$ - pressão de operação do aspersor, $\mathrm{kPa}$, e

DB - diâmetro do bocal, mm.

O produto da lâmina média aplicada, considerada igual à irrigação total necessária (ITN), pela eficiência de aplicação em potencial $\left(E A_{P}\right)$ para cada valor de CUC, resultou na estimativa da lâmina média coletada nos 36 pluviômetros da área $(\mathrm{Lm})$ ao longo do ciclo da cultura (eq.(11)). Os valores estimados das lâminas totais coletadas em cada coletor (w), para cada valor de CUC preestabelecido, foram calculados mediante a multiplicação do valor da lâmina relativa coletada em cada pluviômetro, obtida por meio da análise de distribuição normal, com o valor estimado da lâmina média coletada $(\mathrm{Lm})$.

$$
\mathrm{Lm}=\mathrm{ITNEA}_{\mathrm{p}}
$$

em que,

Lm - estimativa da lâmina média coletada nos 36 pluviômetros, mm;

ITN - irrigação total necessária, mm, e

$\mathrm{EA}_{\mathrm{p}}$ - eficiência de aplicação em potencial.

Com base na função de produtividade, gerada a partir dos dados encontrados por FIGUERÊDO et al. (2008), e utilizando-se dos valores estimados de lâmina coletada, em cada pluviômetro, durante todo o período vegetativo da cultura (w), foi calculada a produtividade de grãos do feijoeiro, em $\mathrm{kg} \mathrm{ha}^{-1}$, para cada área unitária de influência do pluviômetro coletor (3 $33 \mathrm{~m})$.

Determinou-se uma função relacionando a produtividade e a uniformidade de aplicação da água do sistema de irrigação por aspersão convencional, considerando a variação da uniformidade 
de aplicação da água no solo, no cálculo da produtividade da cultura, em função da variação das lâminas de irrigação aplicadas.

\section{RESULTADOS E DISCUSSÃO}

Na Tabela 1, são apresentados os valores das lâminas e eficiências do sistema nos diferentes valores de CUC avaliados, observando-se que a lâmina de irrigação sazonal requerida pela cultura foi de $379,65 \mathrm{~mm}$. A eficiência de aplicação em potencial (EAp), que reflete as perdas por evaporação e arrastamento pelo vento, apresentou um valor de 84,88\%. Esse valor, juntamente com a eficiência de distribuição $\left(\mathrm{ED}_{80}\right)$, que reflete as perdas por percolação, permitiu o cálculo da eficiência de aplicação $\left(\mathrm{EA}_{80}\right)$ do sistema de aspersão, para cada valor de coeficiente de uniformidade (CUC) avaliado.

TABELA 1. Valores das lâminas de irrigação e eficiências do sistema nos diferentes valores de CUC (Coeficiente de Uniformidade de Christiansen) avaliados. Values of irrigation depth and system efficiencies at different values of CUC (Christiansen Uniformity Coefficient) evaluated.

\begin{tabular}{|c|c|c|c|c|c|c|}
\hline & \multicolumn{6}{|c|}{ CUC (\%) } \\
\hline & 65 & 70 & 75 & 80 & 85 & 90 \\
\hline Irrigação real necessária IRN (mm) & 379,65 & 379,65 & 379,65 & 379,65 & 379,65 & 379,65 \\
\hline $\begin{array}{l}\text { Eficiência de aplicação em potencial } \\
\text { EAp (decimal) }\end{array}$ & 0,849 & 0,8 & 0,849 & 0,849 & 0,849 & 0,8 \\
\hline Eficiência de distribuição $\mathrm{ED}_{80}(\%)$ & 61,05 & 73,69 & 75,34 & 79,41 & 84,16 & 90,79 \\
\hline Eficiência de aplicação $\mathrm{EA}_{80}(\%)$ & 51,82 & 62,55 & 63,95 & 67,40 & 71,44 & 77,06 \\
\hline Irrigação total necessária ITN (mm) & 732,64 & 606,97 & 593,68 & 563,25 & 531,46 & 492,65 \\
\hline Lâmina média coletada Lm (mm) & 621,87 & 515,20 & 503,92 & 478,09 & 451,11 & 418,16 \\
\hline
\end{tabular}

Devido às menores perdas por percolação em sistemas com melhor uniformidade de aplicação da irrigação, os maiores valores de eficiência de aplicação foram encontrados para os maiores valores de CUC. Verificou-se, também, que o valor da lâmina média coletada (Lm) nos pluviômetros, no ciclo da cultura, foi menor à medida que o sistema apresentava maiores valores de CUC. A lâmina média aplicada (ITN) variou de 492,65 $\mathrm{mm}$ até 732,64 $\mathrm{mm}$ para valores de CUC iguais a 90 e $65 \%$, respectivamente, o que representa uma economia de água de $240 \mathrm{~mm}$ ou 32,8\% no ciclo da cultura, caso haja elevação da uniformidade de aplicação de água do sistema.

Na Figura 1, é possível observar os perfis de distribuição de água do sistema para os diferentes valores de CUC avaliados. A linha horizontal, que cruza o "eixo y" sobre o valor da IRN, permite observar as áreas de percolação (P) e de déficit (D). Observa-se que $80 \%$ da área, definida como a percentagem de área adequadamente irrigada, apresentaram lâmina maior ou igual à IRN $(379,65 \mathrm{~mm})$. Verificou-se também, à medida que o coeficiente de uniformidade aumentou, que as menores valores foram as perdas por percolação e as áreas de déficit, sendo isto observado pela menor inclinação do perfil de distribuição para maiores valores de CUC. 
CUC $65 \%$

Área irrigada $(\%)$

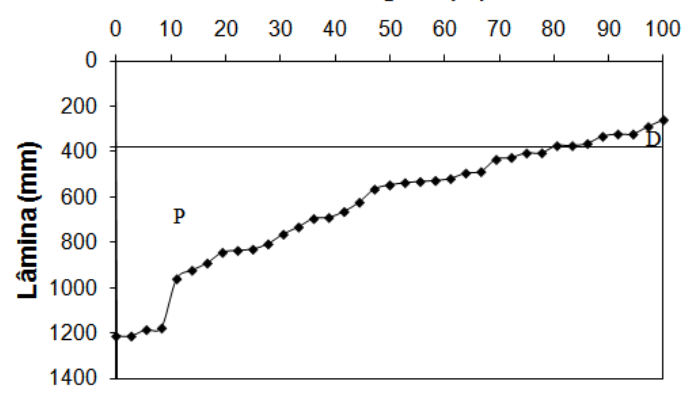

CUC $75 \%$

Área irrigada (\%)

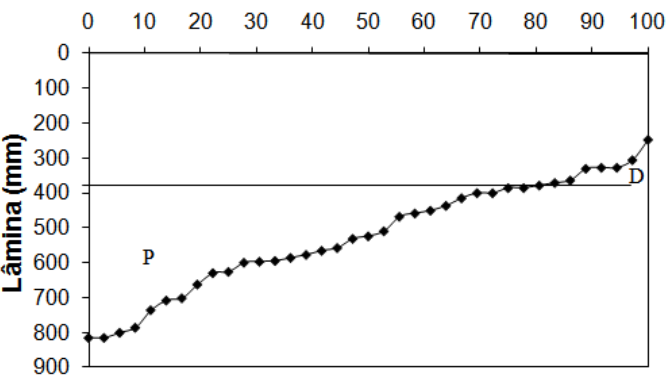

CUC $85 \%$

Área irrigada (\%)

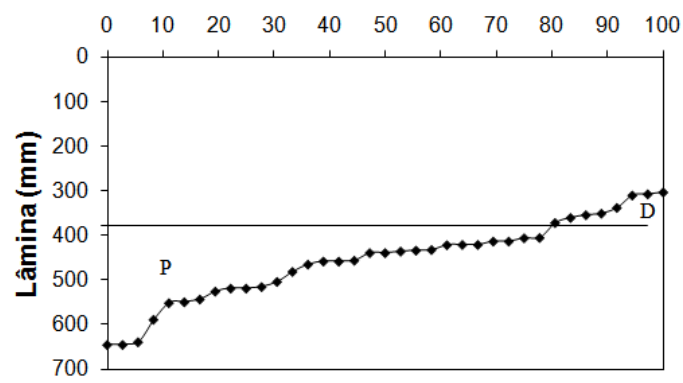

CUC $70 \%$

Área irrigada $(\%)$

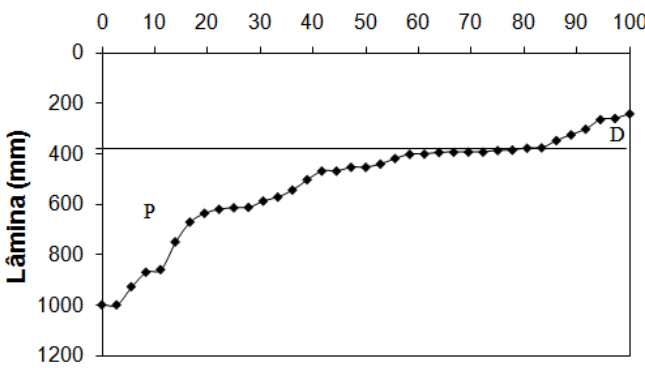

CUC $80 \%$

Área irrigada $(\%)$

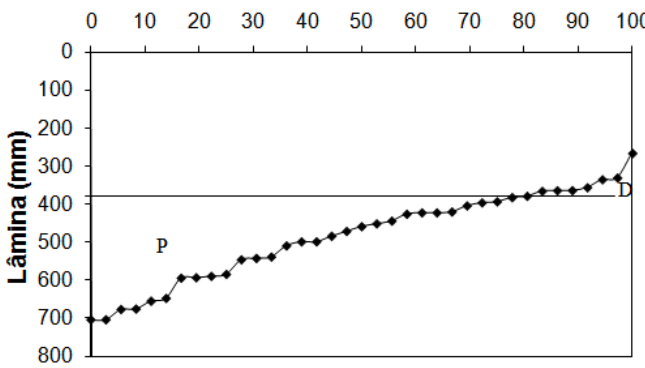

CUC $90 \%$

Área irrigada (\%)

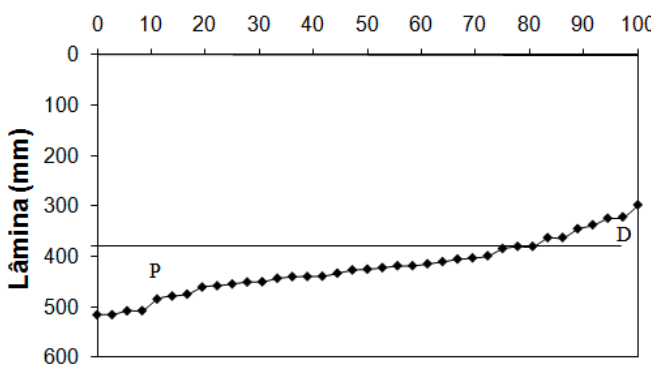

FIGURA 1. Perfis de distribuição de água para os diferentes valores de CUC avaliados. Water distribution pattern for different evaluated CUC values.

Com relação aos valores de produtividade obtidos em função da uniformidade de aplicação do sistema de irrigação (Tabela 2), observou-se aumento na produtividade de grãos mediante o aumento dos valores de CUC. A produtividade de grãos para um CUC de $90 \%$ e $65 \%$ apresentou valores de $2.946,52 \mathrm{~kg} \mathrm{ha}^{-1}$ e $1.974,98 \mathrm{~kg} \mathrm{ha}^{-1}$, respectivamente, apresentando uma diferença de $971,54 \mathrm{~kg} \mathrm{ha}^{-1}$, sendo possível constatar a importância da uniformidade de aplicação de água no rendimento da cultura. Isso ocorre porque, quanto menor a uniformidade de irrigação, maior será o excedente de irrigação nas áreas superirrigadas e maior também será o déficit de água para a cultura, causando redução de produtividade pelo encharcamento do solo e estresse hídrico, respectivamente. As maiores diferenças na produtividade foram observadas com os menores valores de CUC (65 e $70 \%$ ). O inverso foi notado para os maiores níveis de CUC (85 e 90\%), que apresentaram os respectivos rendimentos: $453,53 \mathrm{~kg} \mathrm{ha}^{-1}$ e $51,45 \mathrm{~kg} \mathrm{ha}^{-1}$, devido à maior amplitude entre os valores máximo e mínimo unitários de lâmina coletada. 
TABELA 2. Produtividade da cultura para os diferentes valores de CUC do sistema. Crop yield for different CUC values of the system.

\begin{tabular}{lcccccc}
\hline \multicolumn{7}{c}{ CUC $(\%)$} \\
\hline Produtividade $\left(\mathrm{kg} \mathrm{ha}^{-1}\right)$ & 65 & 70 & 75 & 80 & 85 & 90 \\
\hline
\end{tabular}

Na Figura 2, são apresentados a curva e o modelo (polinômio de segundo grau), que relacionam produtividade de grãos com os valores de CUC do sistema. Analisando-se o ajuste quadrático obtido, pôde-se deduzir que o ponto equivalente à máxima produtividade de grãos de feijão foi de 2.946,52 $\mathrm{kg} \mathrm{ha}^{-1}$, correspondendo a um CUC igual a 90\%, demonstrando a importância de uma boa uniformidade na aplicação da água de irrigação no rendimento da cultura.

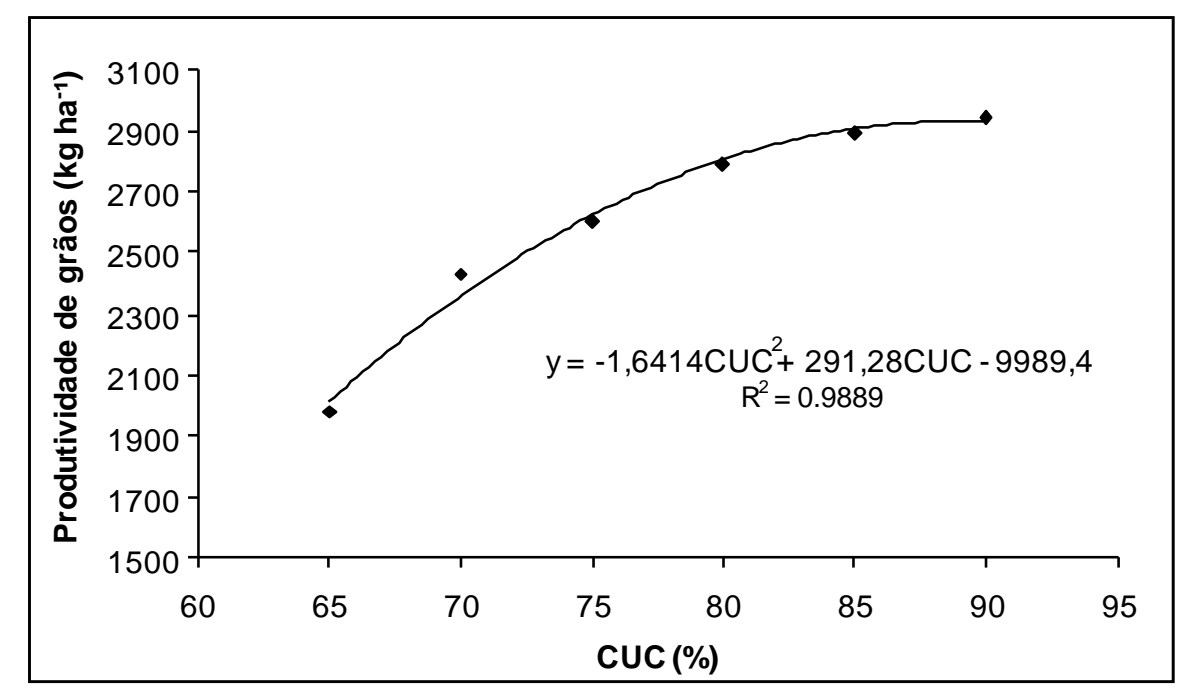

FIGURA 2. Curva de produtividade de grãos em função do CUC para uma lâmina líquida adequada. Grain yield curve as function of the CUC for an adequate net depth.

\section{CONCLUSÕES}

A maior produtividade de grãos $\left(2.946,52 \mathrm{~kg} \mathrm{ha}^{-1}\right)$ foi obtida com o maior coeficiente de uniformidade de aplicação da água do sistema de irrigação por aspersão (90\%), que resultou no menor valor de lâmina média coletada $(418,16 \mathrm{~mm})$ no ciclo da cultura do feijoeiro.

O modelo e o método adotados mostraram-se adequados para gerar cenários de produtividade do feijoeiro para as condições ambientais da região de estudo.

\section{REFÊRENCIAS}

BERNARDO, S. Irrigação: total, suplementar, com déficit e de salvação. Revista ITEM, Brasília, n.71/72, p.64-68, 2006.

BERNARDO, S.; SOARES, A.A.; MANTOVANI, E.C. Irrigação por aspersão. In: Manual de irrigação. 8.ed. Viçosa: UFV2008. p.361-482.

BINOTTI, F.F.S.; ARF, O.; ROMANINI JÚNIOR, A.; FERNANDES, F.A.; SÁ, M.E.; BUZETTI, S. Manejo do solo e da adubação nitrogenada na cultura de feijão de inverno e irrigado. Bragantia, Campinas, v.66, n.1, p.121-129, 2007.

DOORENBOS, J.; KASSAM, A. H. Efectos del agua sobre el rendimiento de los cultivos. Roma: FAO, 1979. (Riegos y Drenaje, 33). 
EFETHA, A.; HARMS, T.; BANDARA, M. Irrigation management practices for maximizing seed yield and water use efficiency of Othello dry bean (Phaseolus vulgaris L.) in Southern Alberta, Canada. Irrigation Science, New York, v.29, p.103-113, 2011.

FARIA, L.C.; COLOMBO, A.; OLIVEIRA, H.F.E.; PRADO, G. do. Simulação da uniformidade da irrigação de sistemas convencionais de aspersão operando sob diferentes condições de vento. Engenharia Agrícola, Jaboticabal, v.29, n.1, p.19-27, 2009.

FIGUERÊDO, S.F.; POZZEBON, E.J.; FRIZZONE, J.A.; AZEVEDO, J.A.; GUERRA, A.F.; SILVA, E.M. Gerenciamento da irrigação do feijoeiro baseado em critérios técnicos e econômicos no cerrado. Irriga, Botucatu, v.13, n.3, p.378-391, 2008.

FREITAS, P.S.L.; REZENDE, R.; MANTOVANI, E.C.; FRIZZONE, J.A. Viabilidade de inserção dos efeitos da uniformidade de irrigação em modelos de crescimento de culturas. Revista Brasileira de Engenharia Agrícola e Ambiental, Campina Grande, v.7, n.3, p.437-444, 2003.

FRIZZONE, J.A.; REZENDE, R.; GONÇALVES, A.C.A.; HEBEL JÚNIOR, A. Produtividade do feijoeiro sob diferentes uniformidades de distribuição de água na superfície e na subsuperfície do solo. Engenharia Agrícola, Jaboticabal, v.27, n.2, p.414-425, 2007.

HSIAO, T.C.; STEDUTO, P.; FERERES, E. A systematic and quantitative approach to improve water use efficiency in agriculture. Irrigation Science, n.25, p.209-231, 2007.

JENSEN, M.E. Beyond irrigation efficiency. Irrigation Science, New York, n.25, p.233-245, 2007.

KELLER, J.; BLIESNER, R.D. Sprinkle and trickle irrigation. New York: Van Nostrand Reinold, 1990. $652 \mathrm{p}$.

LIMA, E.P.; SILVA, E.L. da. Temperatura-base, coeficientes de cultura e graus-dia para cafeeiro arábica em fase de implantação. Revista Brasileira de Engenharia Agrícola e Ambiental, Campina Grande, v.12, n.3, p.266-273, 2008.

MANTOVANI, E.C. Determinação do coeficiente de cultura para o feijão (Phaseolus vulgaris L.) irrigado em duas épocas de plantio. 1986. 73 f. Dissertação (Mestrado em Engenharia Agrícola) Universidade Federal de Viçosa, Viçosa - MG, 1986.

MANTOVANI, E.C.; BERNARDO, S.; PALARETTI, L.F. Irrigação - princípios e métodos. 3.ed. Viçosa: Editora UFV, 2009a. 355 p.

MANTOVANI, E.C.; FACCIOLI, G.G.; LEAL, B.G.; SOARES, A.A.; COSTA, L.C.; FREITAS, P.S.L. influence of the water distribution uniformity and irrigation depth on the yield of irrigated bean crop. Irriga, Botucatu, v.14, n.4, p.458-469, 2009 b.

MANTOVANI, E.C.; VILLALOBOS, F.J.; ORGAZ, F.; FERERES, E. Modelling the effects of sprinkler irrigation. Agricultural Water Management, Amsterdam, v.27, n.3, p.243-257, 1995.

MENDONÇA, J.C.; SOUSA, E.F.; BERNARDO, S.; SUGAWARA, M. T.; PEÇANHA, A.L.; GOTTARDO, R.D. Determinação do coeficiente cultural (Kc) do feijoeiro (Phaseolus vulgaris L.), em Campos dos Goytacazes - RJ. Revista Brasileira de Engenharia Agrícola e Ambiental, Campina Grande, v.11, n.5, p.471-475, 2007.

MUNOZ-PEREA, C.G.; ALLEN, R.G.; WESTERMANN, D.T.; WRIGHT, J.L.; SINGH, S.P. Water use efficiency among dry bean landraces and cultivars in drought-stressed and non-stressed environments. Euphytica, Wageningen, n.155, p.393-402, 2007.

OLIVEIRA, F.G.; FIGUEIREDO, F.P. Influência do espaçamento entre aspersores e do uso de válvulas reguladoras de pressão na uniformidade de irrigação por aspersão convencional. Revista Ceres, Viçosa-MG, v.54, n.316, p.506-510, 2007.

PEREIRA, L.S.; VALERO, J.A. de J.; BUENDÍA, M.R.P.; MARTÍN-BENITO, J.M.T. El riego y sus tecnologías. Albacete: CREA-UCLM, 2010. 296 p. 
REZENDE, R.; FREITAS, P.S.L.; MANTOVANI, E.C.; FRIZZONE, J.A. Função de produção da cultura do milho e do feijão para diferentes lâminas e uniformidade de aplicação de água. Acta Scientiarum Agronomy, Maringá, v.26, n.4, p.503-511, 2004.

ROCHA, E.M.M.; COSTA, R.N. T.; MAPURUNGA, S.M.S.; CASTRO, P. T. Uniformidade de distribuição de água por aspersão convencional na superfície e no perfil do solo. Revista Brasileira de Engenharia Agrícola e Ambiental, Campina Grande, v.3, n.2, p.154-160, 1999.

SANTANA, M.J.; CARVALHO, J.A.; ANDRADE, M.J.B.; BRAGA, J.C.; GERVÁCIO, G.G. Coeficiente de cultura e análise do rendimento do feijoeiro sob regime de irrigação. Irriga, Botucatu, v.13, n.1, p.92-112, 2008.

SANTOS, C.; LORITE, I.J.; TASUMI, M.; ALLEN, R.G.; FERERES, E. Performance of an irrigation scheme using indicators determined with remote sensing techniques. Irrigation Science, New York, n.28, p.461-477, 2010.

SCHMIDT, E.W.; VALIATI, M.I. Avaliação do manejo de irrigação para a cultura de feijão (Phaseolus vulgaris) irrigada por pivô central na Agrícola Wehrmann em Cristalina - GO. Revista Campo Digital, v.1, n.1, p.23-31, 2006. 